\title{
RAPID MAXILLARY EXPANSION AND NASO-PHARYNGEAL AIRWAY A THREE DIMENSIONAL ASSESSMENT
}

\author{
Noha Hussein Abbas*, Walaa Mohamed Hamed ${ }^{* *}$ and Shaimaa Mohamed Abu el Sadat ${ }^{* * *}$
}

\begin{abstract}
The two dimensional plain cephalometric radiographs were widely used to assess the post-rapid maxillary expansion (RME) effect on the naso-pharyngeal airway. However,volumetric changes in the airway need proper assessment. The aim of this study was to assess the linear and volumetric changes in the airway pre and post orthodontic RME using cone beam computed tomography (CBCT). A sample of 12 participants ( 8 males, 4 females with mean age $14 \pm 1.3$ ) who needed RME for the management of posterior crossbite due to transverse maxillary deficiency was used in this study. CBCT scans were acquired pre and post maxillary expansion. Linear and angular measurements were calculated in addition to the volumetric measurements were obtained. There was a statistically significant increase in inter-molar width and molar tipping. The nasal cavity showed statistically significant increase in the width of the nasal floor and the lateral nasal width. There was also statistical significant increase in naso and oropharygeal airway measurements post-expansion. RME using hyrax appliance increased the nasal floor width, nasal lateral width and the airway volume at the naso and oropharyngeal level
\end{abstract}

\section{INTRODUCTION}

Maxillary constriction is the most prevalent facial deformity. It is common to find the maxillary constriction associated with other skeletal abnormalities. ${ }^{1,2}$ The clinical finding of maxillary constriction might be constriction of the palate, dental crowding with decreased arc perimeter, dental rotations and unilateral or bilateral posterior crossbite,. It could also be associated with difficulties in nasal breathing, mouth breathing, and sometimes middle ear problems. ${ }^{3,4}$

Posterior crossbite with its different types is a common malocclusion with a prevalence of $4 \%$ to $23 \% .^{1-3}$ Rapid maxillary expansion (RME) is used to correct posterior crossbite and resolve the problems associated with maxillary constriction where it increases maxillary width, and increase the arch perimeters to relieve crowding. RME was first de-

* Lecturer of Orthodontics, Faculty of Dentistry, Ain Shams University.

** Associate Professor of Oral and maxillo-facial Radiology, Faculty of Dentistry, Ain Shams University.

*** Lecturer of Oral and maxillo-facial Radiology, Faculty of Dentistry, Ain Shams University. 
scribed by Angell ${ }^{5}$ in 1860 and was popularized by Haas 100 years later. ${ }^{6,7}$

The new era is towards more conservative nonextraction treatment and the achievement of broader, more esthetic smiles. This made the RME a frequently used treatment modality to achieve a wide range of orthodontic goals even in the absence of posterior cross bite. ${ }^{6-9}$

Rapid maxillary expansion appliances produce are so constructed to produce heavy forces that separate the maxillary suture, resulting in orthopedic expansion and with minimum tooth movement. ${ }^{6-8}$ Despite concentrated primarily on splitting the maxillary suture, rapid maxillary expansion forces also affect the surrounding frontomaxillary, zygomaticomaxillary, zygomaticotemporal, and pterygopalatine sutures. ${ }^{10}$

As the maxillary bones form half of the anatomic structures of the nasal cavity, it was hypothesized that mid-palatal disjunction will affect nasal cavity anatomy and consequently physiology. ${ }^{11}$

Several studies reported an increase in the width of the nasal cavity which can lead to decreased nasal resistance, improved airflow ${ }^{6,7,12}$ and thus improved nasal breathing. ${ }^{13}$

Several studies evaluated the effect of RME on the skeletal as well as dental structures using various techniques including manual measurement of dental casts and plane film lateral ${ }^{13,14}$ and posteroanterior cephalograms. ${ }^{13,15}$

Similarly, the changes in the nasal and pharyngeal airway spaces after RME have been investigated widely using two dimensional (2D) cephalometric radiographs. ${ }^{16}$ Meanwhile, the three dimensional (3D) anatomy of the airway spaces made it difficult to assess the effects of RME precisely using 2D imaging techniques. The complexity of the airway anatomy is due to the existence of many bony structures The superimposition of bilateral structures and the presence of numerous bony structures, the dif- ficulty in identification of these structures and the superimposition of the bilateral bones. This presents a major obstacle to 2D assessment. Additionally, 2D imaging technique lack information regarding volumetric changes.

Montgomery et al ${ }^{18}$ performed human cadaver studies using computed tomography (CT). They showed that accurate volumetric measurements of the nasal airway can be obtained from CT images. The aim of this study was to assess the linear and volumetric changes in the airway pre and post orthodontic RME using CBCT.

\section{MATERIALS AND METHODS}

This retrospective study was based on pre- and post-treatment CBCT records of 12 participants ( 8 males, 4 females with mean age $14 \pm 1.3$ ) who needed RME for the management of posterior crossbite due to transverse maxillary deficiency. Participants with missing or low quality records or previous airway surgery or craniofacial deformity or those who underwent previous orthodontic treatment were excluded. The patients were informed about the nature of the study and they agreed to participate by signing the informed consents. A hyrax expander supported with acrylic plate was used where the screw was activated quarter turn $(0.25 \mathrm{~mm})$ twice a day till the posterior crossbite was corrected then it was fixed with acrylic resin and kept passive in place for 6 months after which the post-expansion CBCT was taken.

All CBCT examinations were acquired using i-CAT Next Generation (Imaging Sciences International, Hatfield, Pennsylvania, USA). Exposure parameters: $120 \mathrm{kVp}, 0.3 \mathrm{~mm}$ voxel size and 17.8 sec Scanning time with Field of View (FOV) $23 \mathrm{X}$ $17 \mathrm{~cm}$. The head was stabilised by a headband to prevent movement during the scan. The scans were completed with the patients in an upright head position which was adjusted via the laser beams of the machine. The scans were obtained with the tongue 
resting on the palate, swallowing and breathing were evaded during the scan. CBCT scans were acquired twice before and after maxillary expansion. All DICOM (Digital Imaging and Communications in Medicine) files attained in each scan were exported to Invivo 5.3 version Anatomage Invivo software. Measurements were done pre and post maxillary expansion. All measurements were blindly taken via an oral and maxillofacial radiologist and were repeated after one week. To validate the reproducibility, the two sets of measurements were compared. Linear, angular and volumetric measurements were performed in addition to the airway volumetric analysis.

\section{Linear and angular measurements}

\section{Molar tipping and Molar-to-Molar width}

The coronal plane is adjusted so that the apices of the palatal root of both the right and left first molars were visible. The angle formed by the lines traced from the mesiobuccal cusps to the palatal root apices of the right and left molars were measured representing the molar tipping. The distance between the lingual crests of the upper first molar on the same coronal cut was also measured representing molar width.
The nasal floor width (NFW) and nasal lateral width (NLW)

The anterior nasal spine was adjusted to be on the same line with the posterior nasal spine. The distance between the 2 landmarks was measured and the coronal plane was adjusted to pass through half the distance between them. The nasal floor width and the nasal lateral width were both measured on the same coronal cut Fig (1).

\section{Volumetric measurements}

The airway measurement icon in the volume render screen was used to measure the volume of the naso and oropharynx Fig. (2).

\section{Statistical analysis}

All measurements were analyzed using a statistical software package (SPSS version 17.0, Chicago, III) for windows. Normal distribution of the data was confirmed using the KolmogoroveSmirnov test, hence, the student $\mathrm{t}$-test $(\mathrm{P}<0.05)$ was used to compare linear and volumetric changes in the nasopharyngeal spaces. Correlations between the changes were assessed using Pearson Correlation Coefficients (PCC). Reproducibility and reliability of all measurements were undertaken using Dahlberg Error Test (DET) and Interclass Correlation Coefficient (ICC).

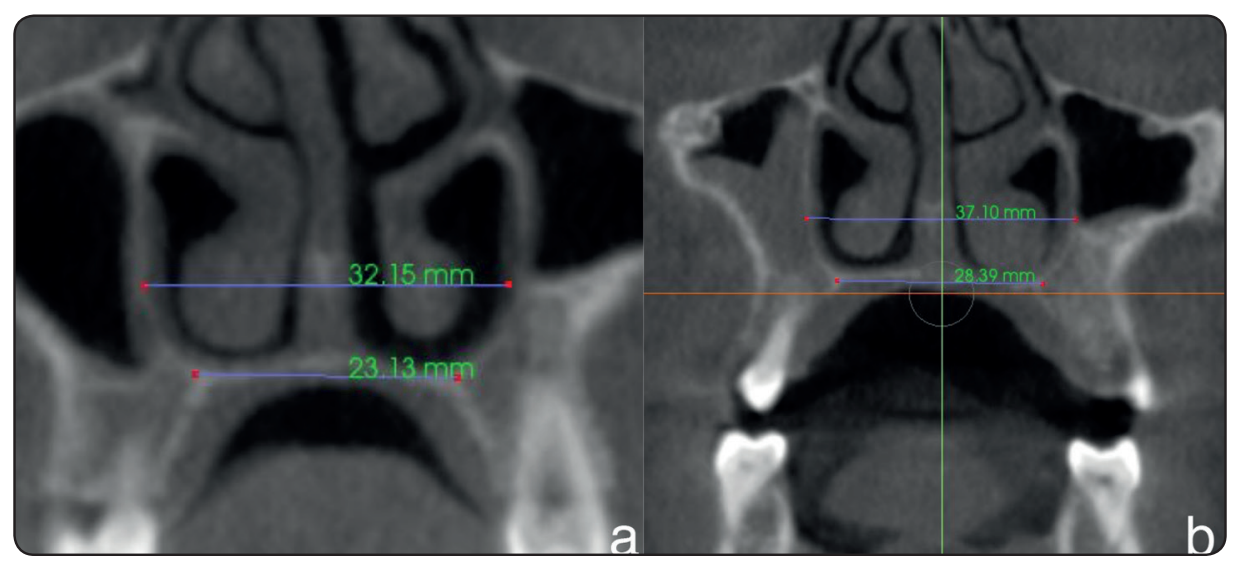

Fig. (1) Measurements of the nasal floor width (NFW) and nasal lateral width (NLW) pre and post maxillary expansion showing an increase in both measurements, a. Pre-maxillary expansion, b. Post-maxillary expansion 


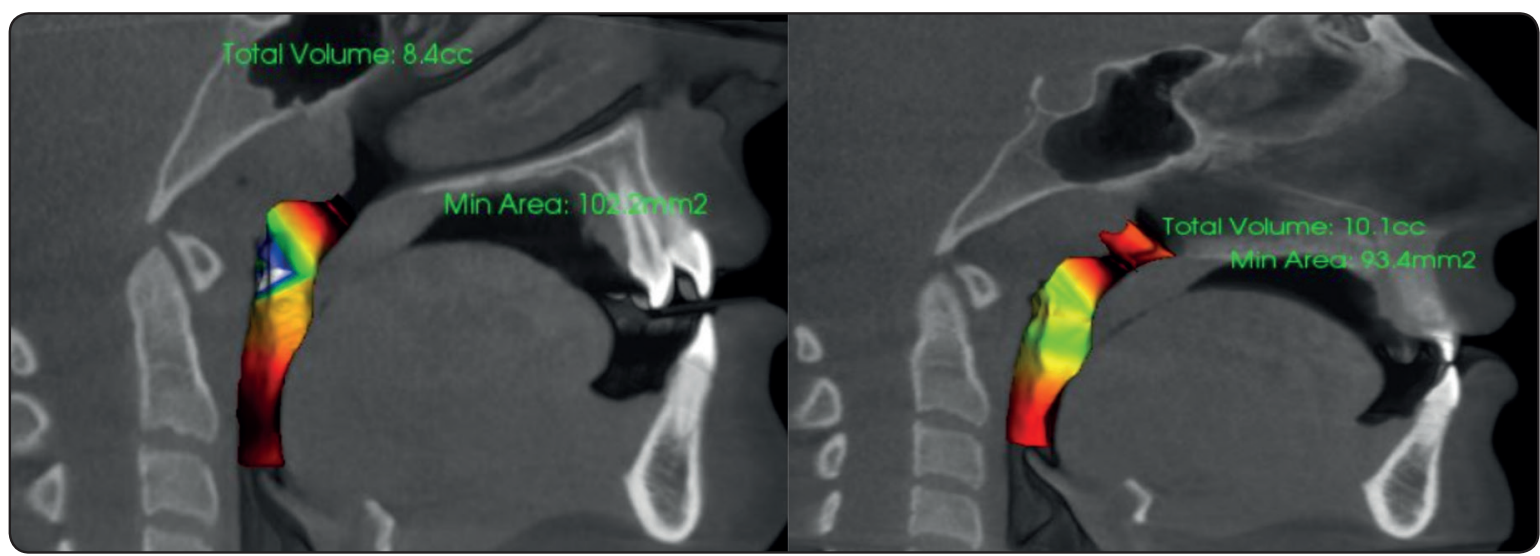

Fig. (2): Volumetric airway measurement pre and post-maxillary expansion displaying a value of 8.4 cc before expansion and $10.1 \mathrm{cc}$ after expansion

TABLE (I) Linear and angular measurements pre and post expansion.

\begin{tabular}{|c|c|c|c|c|c|c|c|c|}
\hline & \multicolumn{2}{|c|}{ Molar to molar width(mm) } & \multicolumn{2}{c|}{ Molar Tipping (deg) } & \multicolumn{2}{c|}{ Nasal floor width (mm) } & \multicolumn{2}{c|}{ Nasal Lateral Width (mm) } \\
\hline & pre & post & pre & post & pre & post & pre & post \\
\hline Mean(SD) & $30(4)$ & $35(3.6)$ & $59(7.3)$ & $70.6(8)$ & $26(4.5)$ & $27.6(4)$ & $33(3)$ & $34.3(3.2)$ \\
\hline P-value & \multicolumn{3}{|c|}{0.000} & \multicolumn{2}{|c|}{0.001} & & 0.003 & 0.003 \\
\hline
\end{tabular}

\section{RESULTS}

Table I shows the linear and angular changes post-expansion. There was a statistically significant increase in inter-molar width and molar tipping. The nasal cavity showed statistically significant increase in nasal floor width and nasal lateral width.

The pre and post volumetric changes are shown in figure 3 . There was a statistically significant increase in naso and oropharygeal airway measurements post-expansion.

\section{DISCUSSION}

RME showed to have an effect on the airway in addition to the effect on the skeletal, dental and soft tissue structures. Moreover miscelleneous effects on hearing, and facial growth were found. ${ }^{1}$ Changes in the pharyngeal airway spaces secondary to RME have been investigated in many studies using two dimensional (2D) cephalometric radiographs. ${ }^{13-15}$

Nowadays, CBCT is widely used to obtain digital data of the anatomy of both the nose and pharynx despite being later introduced than the magnetic resonance imaging (MRI) and computed tomography (CT). The CBCT has privileges of reduced costs and radiation dose over the MRI and CT which justified to the widespread use of this technology. ${ }^{18}$ Thus the aim of the present study was to assess the linear and volumetric changes in the airway and compare pre and post orthodontic RME values using CBCT.

A large field of view (FOV) was used in this study to show all the regions of interest needed for airway analysis. Big voxel sizes produce less radiation as they need less scan and reconstruction time and thus are better used for large FOV's. Nevertheless, large voxel sizes reduce spatial resolution. ${ }^{19}$ 
All DICOM (Digital Imaging and Communications in Medicine) files attained in each scan in this study were exported to Invivo 5.3 version Anatomage Invivo software. Despite the high frequent use of CBCT in dentistry, there are no specified well established protocols for airway analysis. ${ }^{20}$ In their study, El and Palomo ${ }^{21}$ compared three software packages: Dolphin3D, InVivo Dental and OnDemand 3D. They concluded that Dolphin 3D has high reliability, yet poor accuracy. There were also inconsistencies within the softwares themselves. The gray values on CBCT images and the Hounsfield units from multi-slice CT do not correspond. Thus it is not feasible to perform image estimation regarding each tissue. All voxels were put together and their gray values are utilized to render the surface disclosure in order to adjust soft tissue boundaries. ${ }^{22}$

The result of this study showed a significant difference between molar tipping and intermolar distance pre and post RME. RME was initially investigated using CT scanning in a pilot study in the $1980 \mathrm{~s}^{23}$ and further research using this methodology was recommended.

Nasal floor and nasal lateral width showed a significant difference pre and post RME. This is in accordance with Cappellette et $\mathrm{al}^{24}$ who evaluated the skeletal transverse changes of the maxilla and nasal cavity after RME. They concluded that RME produced significant width increases in both, a useful effect that can help in improving respiratory function as well as craniofacial development. Volumetric measurement of airway analysis in this study showed a significant difference between pre and post RME. This could be attributed to lateral displacement of the nasal cavity, namely the outer wall, the nasal conchae and also the inferior turbinates. The lateral bending of the alveolar bone and the inferior nasal floor movement could be an explanation too. While these results are in accordance with the findings of Change et al. ${ }^{25}$ However, Zhao et al. ${ }^{26}$ found no evidence to support the hypothesis that RME treatment increases the volume of the oropharyngeal airway despite the increased intermolar width after RME treatment. Similarly, PangrazioKulbersh et $\mathrm{a}^{27}$ assed the effect of expanders on 23 patients. They reported a dimensional increase at the maxillary sinus level, but no difference in the oropharyngeal airway was detected. The upper limit of the airway was the posterior nasal spine and the epiglottis was the inferior border.

These differences in results between the various studies could be explained by two reasons, first; the time of taking the post expansion records where the edema of the retropalatal tissue, resulting from the trauma for mid palatal suture separation, could have caused a decrease in the airway volume immediately after expansion. The 6 month retention in this study could have resolved this edema and thus showed the increase in airway volume. Second; the inconsistent use of CBCT between studies. When DICOM files are available, it is not wise to overlook the characterization of the nasal cavity in addition to the overall volume calculation. The promising increase in nasopharyngeal airway volume post expansion might lead to a reduction in nasal resistance and thus improvement in the nasal breathing. This could be a base for further studies to asses the effect of RME on the management of obstructive sleep apnea. Randomized clinical trials are needed to validate evidence on the role of maxillary expansion on naso-pharyngeal airway morphology. Moreover, the stability of the effects of RME need additional evidence.

\section{CONCLUSION}

From this study we can conclude that:

1- RME using hyrax appliance increased the nasal floor width and nasal lateral width.

2- RME using hyrax appliance increased the airway volume at the naso and oropharyngeal level. 


\section{REFERENCES}

1. Chiari S, Romsdorfer P, Swoboda H, Bantleon H-P, Freudenthaler J. Effects of rapid maxillary expansion on the airways and earsda pilot study. Eur J Orthod 2009;3:135e41.

2. Wertz RA. Skeletal and dental changes accompanying rapid mid- palatal suture opening. Am J Orthod 1970; 58:41-66.

3. Schu“tz-FranssonU,KurolJ. Rapid maxillary expansion effects on nocturnal enuresis in children: a follow-up study. Angle Orthod J 2008;78:201e8.

4. Ong S, Khambay B, McDonald J, Cross D, Brocklebank $\mathrm{L}, \mathrm{Ju} \mathrm{X}$. The novel use of three-dimensional surface models to quantify and visualise the immediate changes of the mid- facial skeleton following rapid maxillary expansion. Surg J 2015;13:132e8.

5. Angell EC. Treatment of irregularities of the permanent or adult teeth. Dent Cosmos 1860;1:540-4.

6. Haas AJ. Rapid expansion of the maxillary dental arch and nasal cavity by opening the mid palatal suture. Angle Orthod 1961;31: 73-89.

7. Haas AJ. Palatal expansion: just the beginning of dentofacial or- thopedics. Am J Orthod 1970;57:219-55.

8. Isaacson RJ, Ingram AH. Forces produced by rapid maxillary ex- pansion. Part II. Forces present during treatment. Angle Orthod 1964;34:261-9.

9. Adkins MD, Nanda RS, Currier GF. Arch perimeter changes on rapid palatal expansion. Am J Orthod Dentofacial Orthop 1990;97:194-9.

10. Starn bach H, Bayne D, Cleall J, Subtelny JD. Facioskeletal and dental changes resulting from rapid maxillary expansion. Angle Orthod 1966;36:152-64.

11. Warren DW, Hershey GH, Turvey TA, Hinton VA, Hairfield WM. The nasal airway following maxillary expansion. Am J Orthod Dentofacial Orthop 1987;91:111-6.

12. Garib DG, Henriques JFC, Janson G, Freitas MR, Coelho RA. Rapid maxillary expansion-tooth tissue-borne versus tooth-borne ex- panders: a computed tomography evaluation of dentoskeletal ef- fects. Angle Orthod 2005;75:548-57.

13. Basciftci FA, Mutlu N, Karaman AI, Malkoc S, Kucukkolbasi $\mathrm{H}$. Does the timing and method of rapid maxillary expansion have an effect on the changes in the nasal dimensions? Angle Orthod 2002;72:118-23.

14. Sarver DM, Johnston MW. Skeletal changes in vertical and anterior displacement of the maxilla with bonded rapid palatal expansion appliances. Am J Orthod Dentofacial Orthop 1989;95:462-6.
15. Asanza S, Cisneros GJ, Nieberg LG. Comparison of hyrax and bonded expansion appliances. Angle Orthod 1997;67:15-22.

16. Cross DL, McDonald JP. Effect of rapid maxillary expansion on skeletal, dental, and nasal structures: a postero-anterior cephalometric study. Eur J Orthod 2000;22:519-28.

17. Montgomery WM, Vig PS, Staub EU, Matteson SR. Computed tomography: a three dimensional study of the nasal airway. Am J Orthod 1979;76:363-75.

18. G. Di Carlo, A. Polimeni, B. Melsen, and P. M. Cattaneo, "The relationship between upper airways and craniofacial morphology studied in 3D. A CBCT study," Orthodontics and Craniofacial Research, vol. 18, no. 1, pp. 1-11, 2015.

19. Da Silveira PF, Fontana MP, Oliveira HW, et al. CBCTbased volume of simulated root resorption - influence of FOV and voxel size. Int Endod J. 2015;48(10):959-965.

20. Horner K, O’Malley L, Taylor K, Glenny AM. Guidelines for clinical use of CBCT: a review. Dentomaxillofac Radiol. 2015;44(1):20140225.

21. El. H Palomo JM. Measuring the airway in 3 dimensions: A reliability and accuracy study. Am J Orthod Dentofacial Orthop 2010;137:S50.e1-S50.e9.

22. Pauwels R, Araki K, Siewerdsen JH, Thongvigitmanee SS. Technical aspect of dental CBCT: state of the art. Dentomaxillofac Radiol. 2015;44(1):20140224.

23. Timms DJ,Preston CB, Daly PF. A computed tomographic assessment of maxillary movement induced by rapid expansion-a pilot study. European Journal of Orthodontics 1982, vol.4 pg123-127.

24. Cappellette M Jr1, Nagai LHY2, Gonçalves RM2, Yuki AK2, Pignatari SSN1, Fujita RR1. Skeletal effects of RME in the transverse and vertical dimensions of the nasal cavity in mouth-breathing growing children. Dental Press J Orthod. 2017;22(4):61-69.

25. Chang Y, Koenig LJ, Pruszynski JE, Bradley TG, Bosio JA, Liu D. Dimensional changes of upper airway after rapid maxillary expansion: a prospective cone-beam computed tomography study. Am J Orthod Dentofac Orthop 2013;143:462e70.

26. Zhao Y., Nguyen M., Gohl E., Mah J. K., Sameshima G., Enciso R. Oropharyngeal airway changes after rapid palatal expansion evaluated with cone-beam computed tomography. American Journal of Orthodontics and Dentofacial Orthopedics. 2010;137(4):S71-S78.

27. Pangrazio-Kulbersh V., Wine P., Haughey M., Pajtas B., Kaczynski R. Cone beam computed tomography evaluation of changes in the naso-maxillary complex associated with two types of maxillary expanders. Angle Orthodontist. 2012;82(3):448-457. 